\title{
Cholesterol Curves to Identify Population Norms by Age and Sex in Healthy Weight Children
}

\author{
Asheley Cockrell Skinner, $\mathrm{PhD}^{1}$, Michael J. Steiner, $\mathrm{MD}^{1}$, Arlene E. Chung, MD, MHA ${ }^{1}$, and \\ Eliana M. Perrin, MD, MPH ${ }^{1}$ \\ ${ }^{1}$ The University of North Carolina at Chapel Hill, Chapel Hill, NC, USA
}

\section{Abstract}

Objective-Develop clinically applicable charts of lipid values illustrating fluctuations throughout childhood and by sex among healthy weight children.

\begin{abstract}
Methods-The National Health and Nutrition Examination Survey (1999-2008) was used to estimate total cholesterol, high-density lipoprotein (HDL), low-density lipoprotein (LDL), and triglycerides by age and sex in healthy weight children age 3 to 17 years. Using LMS procedures, the authors created smoothed curves demonstrating population-based 50th percentile for age and the 75th and 95th percentiles.
\end{abstract}

Results-The curves were based on 7681 children meeting inclusion criteria. Total cholesterol, HDL, and LDL demonstrated peaks at approximately 8 to 12 years for boys. Similar peaks were evident for girls at slightly younger ages, approximately 7 to 11 years. Triglycerides showed peaks for girls, but values were similar across ages for boys.

Conclusions-The use of fixed lipid value cutoffs in established guidelines regardless of age or sex likely mislabels many children as abnormal. The authors' charts may allow for a more nuanced interpretation based on population norms.

\section{Keywords}

lipids; cholesterol; obesity; screening; preventive care

\section{Introduction}

\begin{abstract}
Serum lipid values vary by age through childhood and are also affected by sex, race, and other factors. ${ }^{1-4}$ Recently, Cook et al ${ }^{1}$ published population-based lipid distributions with curves throughout childhood, which reinforced that there are variations in lipid values, particularly around ages 8 to 14 . By tracking serum cholesterol values through childhood and graphing them, the charts clearly demonstrate that a static cutoff of normal and abnormal, as currently recommended, ${ }^{2}$ is inappropriate based on the dynamic physiology of children at different ages. Currently, the American Academy of Pediatrics recommends screening for lipid abnormalities in all overweight children from after age 2 years to no later than 10 years of age and that those with abnormal values should be treated for hypercholesterolemia, initially with lifestyle changes, followed by statin treatment for those over age 8 , should lifestyle changes fail. ${ }^{2}$ These current definitions of normal are based on
\end{abstract}

(C) The Author(s) 2012

Corresponding Author: Asheley Cockrell Skinner, UNC School of Medicine, 231 MacNider, CB 7225, Chapel Hill, NC 27599, USA, asheley@unc.edu.

Declaration of Conflicting Interests

The authors declared no potential conflicts of interest with respect to the research, authorship, and/or publication of this article. 
single cutoff values for children, regardless of age and gender. Thus, the use of sex-stratified "cholesterol curves" based on a population of healthy weight children and adolescents could enhance the interpretation of isolated total cholesterol (TC) or subcomponent values and help guide clinical decision-making.

Unfortunately, because of the known relationship between excess weight and hyperlipidemia, curves that include the full population of children including the obese, as previous reports have, ${ }^{1,5}$ cannot necessarily be inferred as healthy standards or "normal" and therefore have limited applicability to clinical decision making. Since obesity is known to affect lipid values and obesity rates have increased over the years, population-based cholesterol curves may be inappropriately increased relative to what "normal" or "healthy" TC levels should be through the physiologic changes of childhood. Because of this and the fact that screening usually depends on comparison to a healthy population, curves that demonstrate lipid values in a healthy population could provide useful clinical information to aid in interpretation of cholesterol screening results. Therefore, we sought to develop clinically relevant lipid distribution charts by including only healthy weight children and excluding those with lipid values potentially indicative of familial hypercholesterolemia, which could provide a better understanding of healthy children's distributions of lipid values.

\section{Methods}

This study is based on the repeated cross sections of the National Health and Nutrition Examination Survey (NHANES) from 1999 to 2008. NHANES is a stratified, multistage probability sample of the civilian, non-institutionalized population of the United States. It includes computer-based interviews, an in-home questionnaire on a variety of demographic and health topics, an examination including a thorough physical exam with measured heights and weights, and laboratory measures. ${ }^{6}$

\section{Fasting}

Previous work, including work using a population identical to ours, demonstrates very minimal differences in lipid values in children based on fasting time. ${ }^{7}$ Adequate sample size is critical to the creation of population distribution curves, and limiting only fasting values left far too few children for reliable estimates, particularly for low-density lipoprotein (LDL) and triglycerides among young children. Therefore, we chose to include all children, regardless of fasting status, in our analyses. Examinations of curves limited to children who had fasted for at least 8 hours showed few differences in the patterns across age, but with greater instability in the estimates.

\section{Sample}

We included children ages 3 to 17 years, who had at least 1 of the 4 common lipid measurements available (TC, high-density lipoprotein [HDL], LDL, or triglycerides). We used height and weight as measured during the examination component to calculate body mass index (BMI) and determine percentile, using SAS code developed for that purpose. ${ }^{8}$ Using standard definitions, we included only children who were of healthy weight (BMI 5th to 85th percentile for age and sex). We excluded children with very high values that could indicate familial hyperlipidemia (10 with cholesterol greater than $300 \mathrm{mg} / \mathrm{dL}$, 4 with LDL greater than $200 \mathrm{mg} / \mathrm{dL}$, and 3 with triglycerides greater than $350 \mathrm{mg} / \mathrm{dL}$ ).

\section{Laboratory Methods}

Equipment for lipid analyses varied through the years. In 1999-2004, a Roche Hitachi 704 Analyzer was used, and a Roche Hitachi 717 was used in 2005. A Roche Hitachi 912 was 
used in 2006, and a Roche Modular P in 2007-2008. No adjustments were needed for the changes in equipment. ${ }^{9,10}$

TC was measured enzymatically in serum or plasma for all years of data collection. HDL cholesterol in 1999-2002 was measured using a heparin-manganese precipitation, or direct measurement for children ages 3 to 5, because of small sample volumes. Beginning in 2003, all samples were tested using a direct immunoassay. Although changes in HDL values across time indicate possible laboratory method differences, our focus on age-based differences averaged over the period did not necessitate further adjustments. Triglycerides were measured enzymatically in serum for all years. LDL was calculated from total cholesterol, HDL, and triglycerides as follows: [LDL-cholesterol] = [total cholesterol $]-$ [HDL-cholesterol] - [triglycerides/5].

\section{Statistical Methods}

We used Cole's LMS procedures to create population-based curves of lipid values throughout childhood, ${ }^{11,12}$ which is similar but not identical to those used to create the CDC growth charts. ${ }^{13}$ Although sample sizes of 100 at each age point have been recommended for growth curves, smaller sample sizes can be sufficient. ${ }^{14}$ See Table 1 for the sample sizes at each age for each of the lipid measures. Sample sizes are small, particular among 3- to 5year-old children. This suggests that some caution should be used when interpreting data at these young ages; however, we present data for all age groups. The exception is that we exclude 3-year-old children from our triglyceride analyses because of extreme standard errors in the values at this age.

As opposed to a prepackaged program, we used Stata 11.0 (College Station, TX) to calculate the L, M, and S parameters as described by Cole. ${ }^{11,12}$ All analyses were adjusted for the complex survey design of NHANES and were performed using the survey estimation routines in Stata. This study was deemed exempt from institutional review board review under federal regulation 45 CFR $\$ 46.101($ b) because it used only de-identified secondary data.

\section{Results}

The curves (see Figures 1-4) were based on 7681 children who met inclusion criteria. TC, HDL, and LDL demonstrated peaks at $\sim 8$ to 12 years for boys. Similar peaks were evident for girls, though at slightly younger ages, $\sim 7$ to 11 years. Triglycerides showed peaks for girls, but values were similar across ages for boys.

The curves demonstrate important differences from currently recommended cutoff values. Children around age 9 to 10 at the 95 th percentile have total cholesterol values $\sim 15 \mathrm{mg} / \mathrm{dL}$ higher than younger children or adolescents. Although the 95th percentile is similar to or lower than the static cutoff of $200 \mathrm{mg} / \mathrm{dL}$ for younger children and adolescents, it is clearly higher for children in middle childhood.

\section{Discussion}

Our findings confirm previous findings of differences in lipids throughout childhood. ${ }^{1,4} \mathrm{We}$ extend these findings by using a nationally representative sample of healthy weight children and excluding those with extremely elevated lipid values suggesting familial hyperlipidemia syndromes, to create curves that will help clinicians interpret lipid screening results.

Current established guidelines for lipid screening and treatment use fixed lipid value cutoffs regardless of age or sex and are based on population norms that include children who are 
obese and may have familial hyperlipidemia. This likely mislabels many children as "abnormal" and leads to overtreatment of some children with lipid lowering medications as there are clear lipid variations based on age and sex.

The preadolescent peaks in HDL and LDL values for both girls and boys and for triglyceride values for girls could represent physiologic changes that cause lipid variations, and thus examining a patient's lipid values against these curves could be helpful in guiding lipid management. The differences in appearances between the boys' and girls' percentile values are a good reminder of sex-based differences. While there has been some adult literature examining cholesterol differences by $\operatorname{sex}^{15}$ as well as variation during the menstrual cycle for women, ${ }^{16}$ the pediatric differences may be even more stark.

There are several important limitations of this study. First, we use only cross-sectional data, so we are unable to demonstrate if individual children follow the patterns we show.

However, we use a method comparable to those used to create the clinical growth charts, which also rely on cross-sectional data. Second, we were unable to look at variations by race/ethnicity. Sample size limited our ability to examine additional subpopulations in addition to sex. Although race is related to lipid values, patterns of lipid values may be similar throughout childhood.

Future research is needed to examine whether individual children follow these cholesterol curves over time and to determine appropriate abnormal cut-points for lipid values based on age, sex, and race/ethnicity. Additionally, there is a need to have increased sample size in very young children so we can obtain a better understanding about the distribution of lipid values in this group. Some previous studies have demonstrated that children with abnormal values in childhood often have normal values in adulthood ${ }^{17}$ or even just 3 years later. ${ }^{18}$ One possible explanation for this is the natural variations seen in the population norms we present in this study. It is not known whether these natural variations are detrimental to cardiovascular health, and future studies are needed to elucidate this relationship as well.

\section{Conclusions}

Although we do not delineate for a particular percentile to be defined as abnormal in this study, the ability for providers to clearly see how a child's lipid values compare to other children without known risk factors provides useful additional clinical information that can assist in the care of children after lipid screening. Thus, these charts may allow for a more nuanced interpretation based on population norms for healthy weight children.

\section{Acknowledgments}

Funding

The authors disclosed receipt of the following financial support for the research, authorship, and/or publication of this article: Dr Skinner is currently supported by BIRCWH (K12-HD01441). Dr Perrin is supported by an NIH career development award (K23-HD051817). Dr Chung is supported by an NRSA Primary Care Research award from NIH/HRSA (5T32 PE14001-20).

\section{References}

1. Cook S, Auinger P, Huang TT. Growth curves for cardio-metabolic risk factors in children and adolescents. J Pediatr. 2009; 155(suppl 6):e15-e26. [PubMed: 19732566]

2. Daniels SR, Greer FR. Lipid screening and cardiovascular health in childhood. Pediatrics. 2008; 122:198-208. [PubMed: 18596007] 
3. Freedman DS, Dietz WH, Srinivasan SR, Berenson GS. The relation of overweight to cardiovascular risk factors among children and adolescents: the Bogalusa Heart Study. Pediatrics. 1999; 103(6 pt 1):1175-1182. [PubMed: 10353925]

4. Hickman TB, Briefel RR, Carroll MD, et al. Distributions and trends of serum lipid levels among United States children and adolescents ages 4-19 years: data from the Third National Health and Nutrition Examination Survey. Prev Med. 1998; 27:879-890. [PubMed: 9922071]

5. Jolliffe CJ, Janssen I. Distribution of lipoproteins by age and gender in adolescents. Circulation. 2006; 114:1056-1062. [PubMed: 16940191]

6. National Center for Health Statistics. [Accessed November 9, 2011.] National Health and Nutrition Examination Survey: 1999-2010 survey contents. http://www.cdc.gov/nchs/data/nhanes/survey_content_99_10.pdf

7. Steiner MJ, Skinner AC, Perrin EM. Fasting might not be necessary before lipid screening: a nationally representative cross-sectional study. Pediatrics. 10.1542/peds.2011-0844

8. National Center for Chronic Disease Prevention and Health Promotion. Centers for Disease Control and Prevention. [Accessed December 7, 2005.] A SAS program for the CDC growth charts. http://www.cdc.gov/nccdphp/dnpao/growthcharts/resources/sas.htm

9. National Center for Health Statistics. 2005-2006 Data Documentation, Codebook, and Frequencies. Atlanta, GA: National Center for Health Statistics; 2009.

10. National Center for Health Statistics. 2007-2008 Data Documentation, Codebook, and Frequencies. Atlanta, GA: National Center for Health Statistics; 2009.

11. Cole TJ. Fitting smoothed centile curves to reference data. J Roy Stat Soc a Sta. 1988; 151:385418.

12. Cole TJ, Green PJ. Smoothing reference centile curves: the LMS method and penalized likelihood. Stat Med. 1992; 11:1305-1319. [PubMed: 1518992]

13. Kuczmarski RJ, Ogden CL, Guo SS, et al. 2000 CDC Growth Charts for the United States: methods and development. Vital Health Stat. 2002; 11(246):1-190.

14. Wright EM, Royston P. A comparison of statistical methods for age-related reference intervals. J Roy Stat Soc a Sta. 1997; 160:47-69.

15. The Lipid Research Clinics Program Epidemiology Committee. Plasma lipid distributions in selected North American populations: the Lipid Research Clinics Program Prevalence Study. Circulation. 1979; 60:427-439. [PubMed: 312704]

16. Mumford SL, Schisterman EF, Siega-Riz AM, et al. A longitudinal study of serum lipoproteins in relation to endogenous reproductive hormones during the menstrual cycle: findings from the BioCycle study. J Clin Endocrinol Metab. 2010; 95:E80-E85. [PubMed: 20534764]

17. Magnussen CG, Thomson R, Cleland VJ, Ukoumunne OC, Dwyer T, Venn A. Factors affecting the stability of blood lipid and lipoprotein levels from youth to adulthood: evidence from the Childhood Determinants of Adult Health Study. Arch Pediatr Adolesc Med. 2011; 165:68-76. [PubMed: 21199983]

18. Freedman DS, Wang YC, Dietz WH, Xu JH, Srinivasan SR, Berenson GS. Changes and variability in high levels of low-density lipoprotein cholesterol among children. Pediatrics. 2010; 126:266273. [PubMed: 20643721] 


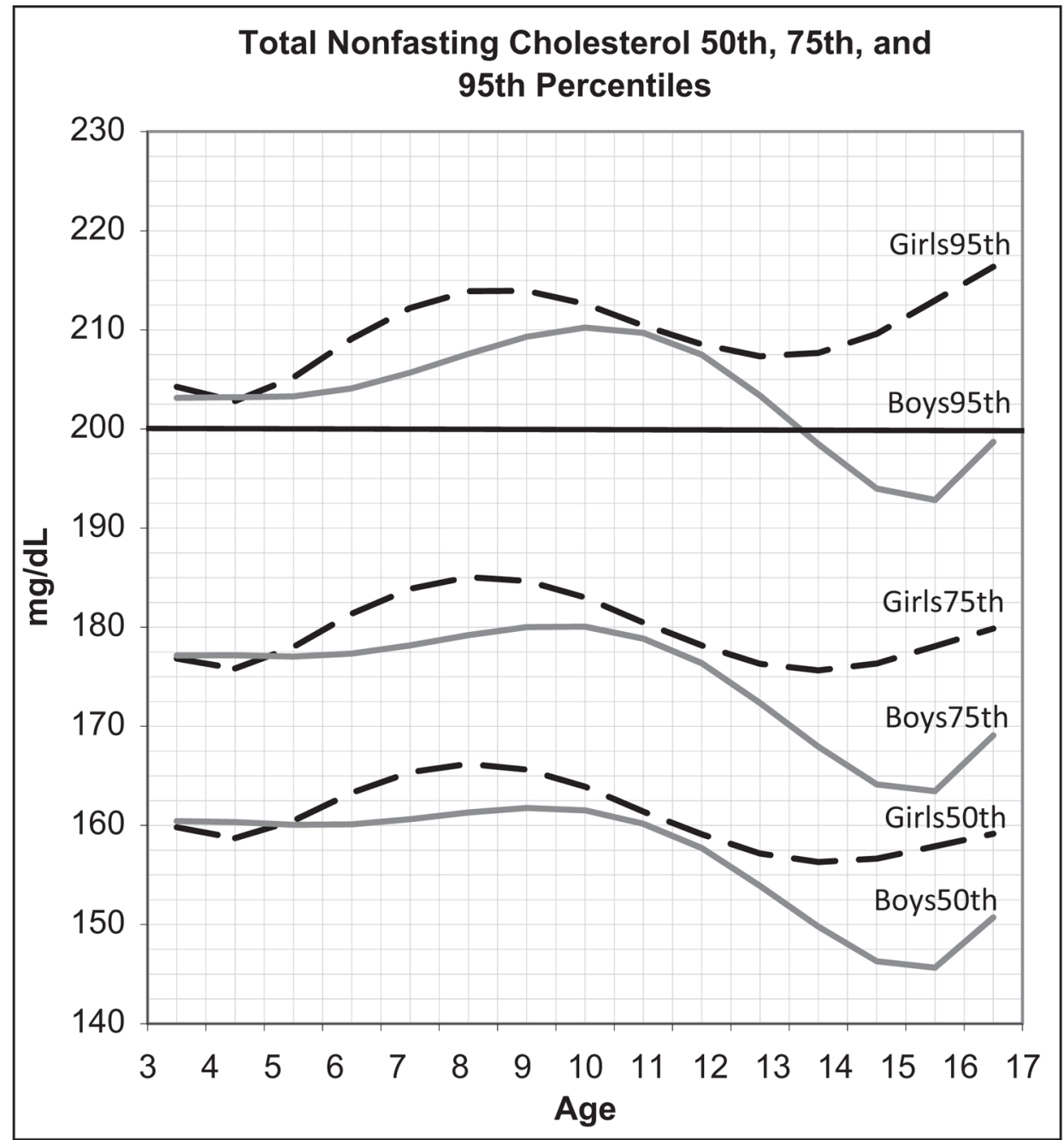

Figure 1.

Distribution of total cholesterol by age

Solid line indicates recommended static cutoff value. 


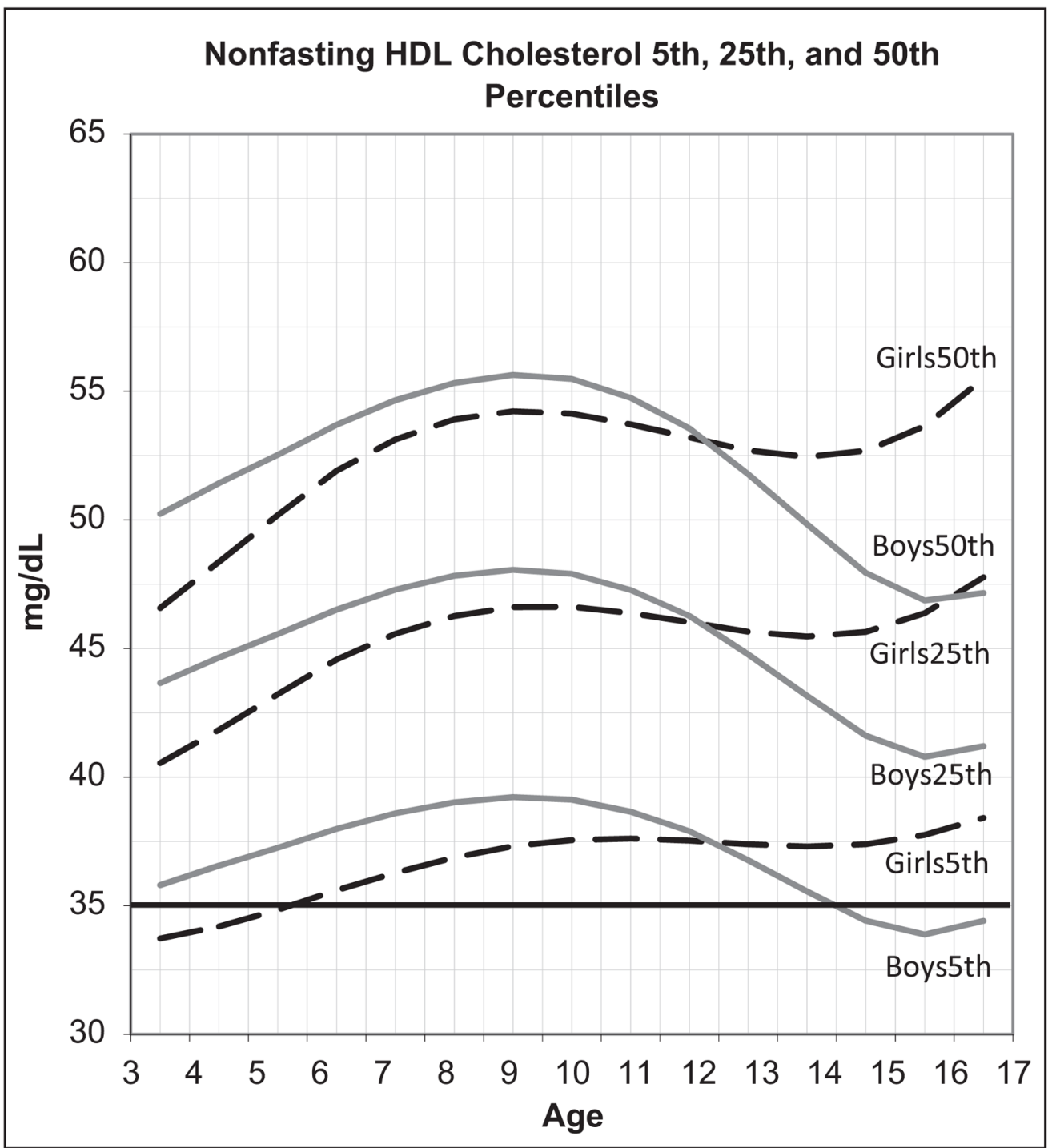

Figure 2.

Distribution of HDL cholesterol by age

Solid line indicates recommended static cutoff value. 


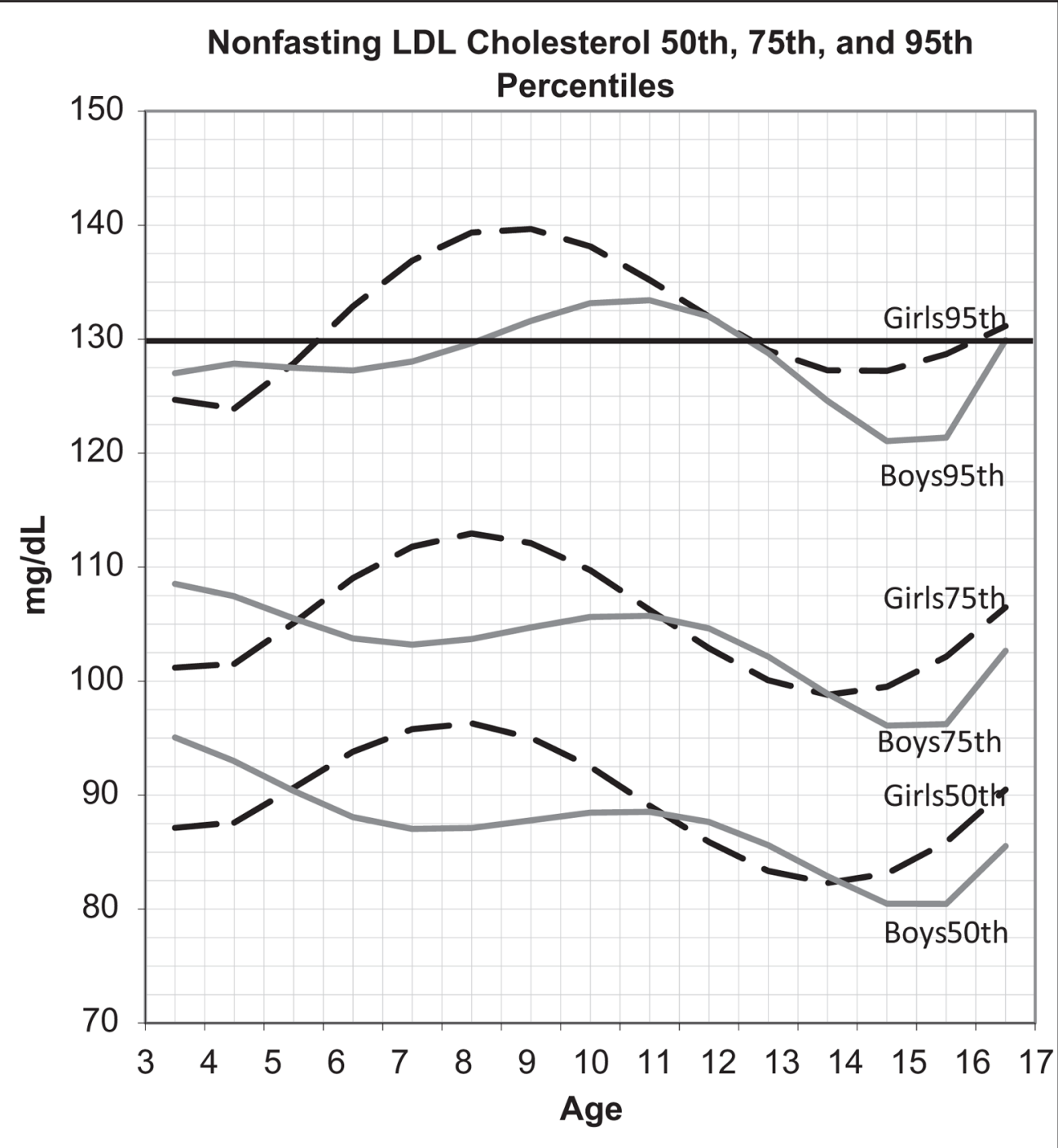

Figure 3.

Distribution of LDL cholesterol by age

Solid line indicates recommended static cutoff value. 


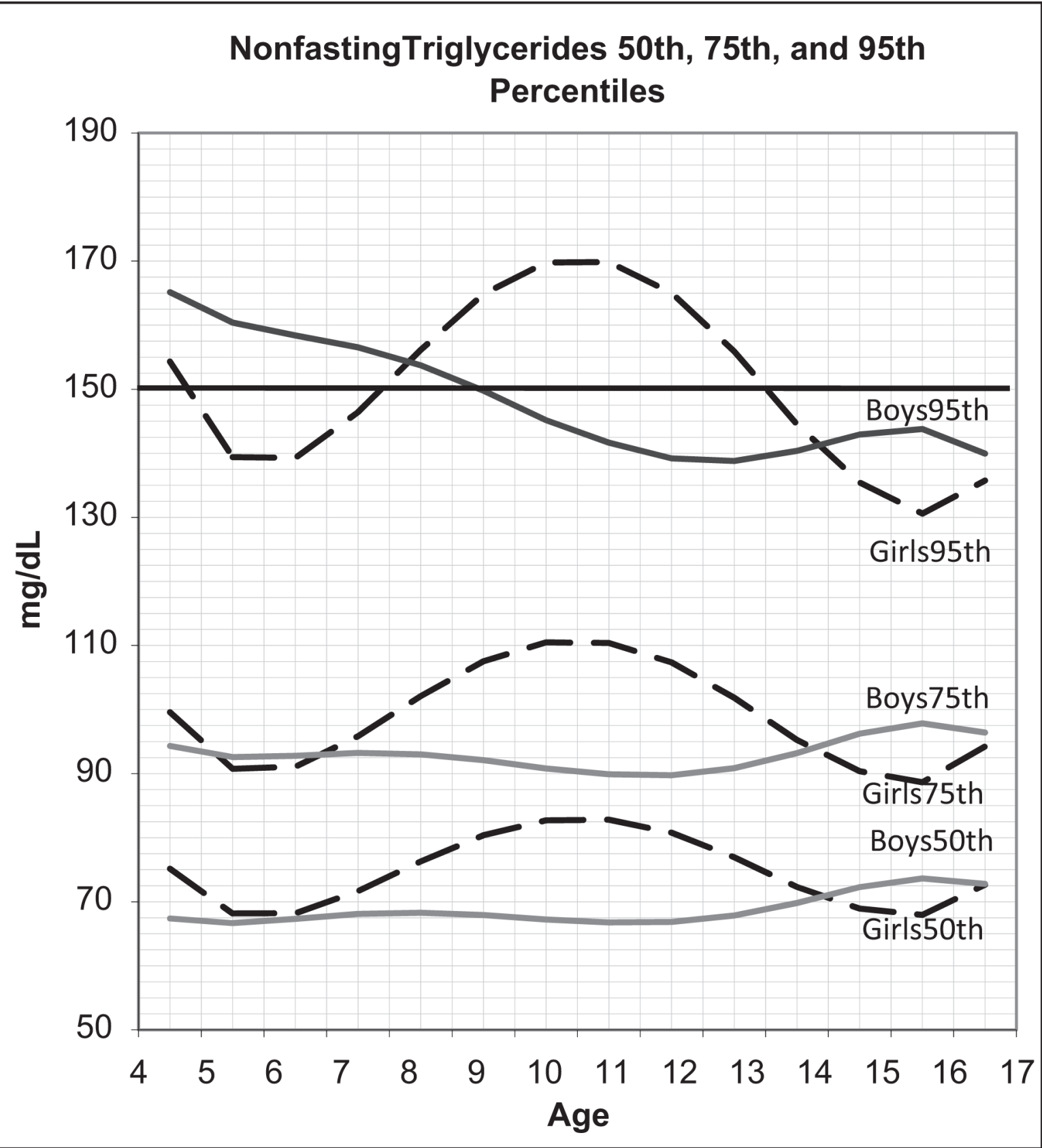

Figure 4.

Distribution of triglycerides by age

Static cut off value is $150 \mathrm{mg} / \mathrm{dL}$. 
